\title{
NOTES
}

\section{Conformation of Amylose and Excluded-Volume Effects on Its Chain Dimensions}

\author{
Yasushi NaKata, ${ }^{\dagger}$ Shinichi Kitamura, Kenichi TaKeo, and Takashi NorisuYE* \\ Department of Agricultural Chemistry, Kyoto Prefectural University, \\ Shimogamo, Kyoto 606, Japan \\ * Department of Macromolecular Science, Osaka University, \\ Toyonaka, Osaka 560, Japan
}

(Received January 19, 1994)

\begin{abstract}
KEY WORDS Amylose / Polysaccharide / Radius of Gyration / Conformation / Simulation / Excluded-Volume Effect / Helical Wormlike Chain /
\end{abstract}

Considerable attention has been paid to dilute-solution behavior of amylose, but our understanding of it still leaves much to be desired. Aqueous solutions of this polysaccharide are notoriously unstable over a broad range in molecular weight $M$ from $8 \times 10^{3}$ to $1.6 \times 10^{5} .^{1,2}$ Because of this unfavorable property, much experimental work $^{3-5}$ so far done could only show that the global conformation of amylose in aqueous solution at room temperature is a random coil having a characteristic ratio $C_{\infty}$ of about 5 at infinite $M$. On the other hand, dimethyl sulfoxide (DMSO) dissolves the polymer of any molecular weight. However, the chain conformation in this organic solvent has long been controversial as to whether it is a random coil or a semiflexible helix. ${ }^{6}$ Conformational energy calculations ${ }^{2,7-9}$ predict that amylose should be flexible but locally helical.

Very recently, Nakanishi et al. ${ }^{10}$ demonstrated from light scattering, sedimentation equilibrium, and viscosity measurements on narrow-distribution samples of synthetic amylose at $25^{\circ} \mathrm{C}$ that the chain conformation in DMSO is a random coil expanded by excluded-volume effect if $M$ is higher than $10^{5}$. They, analyzing intrinsic viscosity $([\eta])$ data for
$M$ between 342 (the dimer) and $10^{4}$ in terms of the unperturbed helical wormlike (HW) chain, ${ }^{11,12}$ derived the following conclusions from the estimated HW model parameters: (1) The value of $C_{\infty}$ in DMSO (at $25^{\circ} \mathrm{C}$ ) is about 5 , so that without excluded-volume effect, the global conformations in DMSO and aqueous solvents are similar, and (2) though flexible, the amylose chain in DMSO has some helical nature locally. This molecular picture, i.e., an irregular helical conformation with high flexibility, appears to resemble that predicted from conformational calculations. ${ }^{2,8,9}$ It is thus intriguing and probably significant to make a quantitative comparison between them in terms of some conformation-dependent property. The most relevant for such a comparison may be the mean-square radius of gyration $\left\langle S^{2}\right\rangle$, for which experimental data ${ }^{10}$ in DMSO are available though limited to high $M$ where excluded-volume effects are significant.

In the present work, we computed $\left\langle S^{2}\right\rangle$ of amylosic chains with or without excluded volume by a Monte Carlo method, and compared the results with the experimental data of Nakanishi et al. ${ }^{10}$ and the $\left\langle S^{2}\right\rangle$ values derived from the HW parameters. The last set

\footnotetext{
${ }^{\dagger}$ Present address: Horiba, Ltd., Kisshoin, Minami-ku, Kyoto 601, Japan.
} 
of $\left\langle S^{2}\right\rangle$ without excluded volume may be taken to reflect the actual viscosity behavior of amylose for $M$ below $10^{4}$ in DMSO. It should also be noted that though the mean-square end-to-end distance $\left\langle R^{2}\right\rangle$ for amylosic chains with or without excluded volume has already been studied by a few groups ${ }^{7-9}$ on the basis of the rotational isomeric state approximation or Monte Carlo methods, the simulation data for $\left\langle S^{2}\right\rangle$ presented below are new.

\section{MONTE CARLO CALCULATIONS}

Amylosic model chains were generated according to the method of Jordan et al. ${ }^{8}$ with the conformational energy map for maltose constructed elsewhere. ${ }^{9}$ The bond angle for the glucosidic oxygen atom was taken as $118^{\circ}$ and the virtual bond length $l$ as $0.44 \mathrm{~nm}$. In computing $\left\langle S^{2}\right\rangle$, each glucose unit was replaced by a hard-core sphere of radius $a$ whose center is located at the center of mass of the glucose unit. This radius represents the magnitude of the excluded-volume repulsion between a pair of glucose residues; no attraction was considered for excluded-volume interactions. Thus, the simulated chains with $a=0$ are unperturbed.

Radii of gyration were obtained for $a=0$, $0.1,0.2,0.3$, and $0.4 \mathrm{~nm}$ over a range of $x$ (the number of glucose residues or virtual bonds) from 2 to 800 . The Monte Carlo sample for each $x$ contained 5000 chains, but for selfavoiding chains with $x>20$, the computation was effected by use of the Wall-Erpenbeck $s-p$ method $^{13}$ of chain enrichment, as in the work by Kitamura et al. ${ }^{9}$ on $\left\langle R^{2}\right\rangle$ (see ref 9 for the details of the procedures). The results all refer to $25^{\circ} \mathrm{C}$. The value of $C_{\infty}$ obtained was 5.0.

The above-mentioned replacement of each glucose unit by a sphere underestimates $\left\langle S^{2}\right\rangle$ by $\Delta$ :

$$
\Delta=\sum_{i} m_{i}\left\langle s_{i}^{2}\right\rangle / \sum_{i} m_{i}
$$

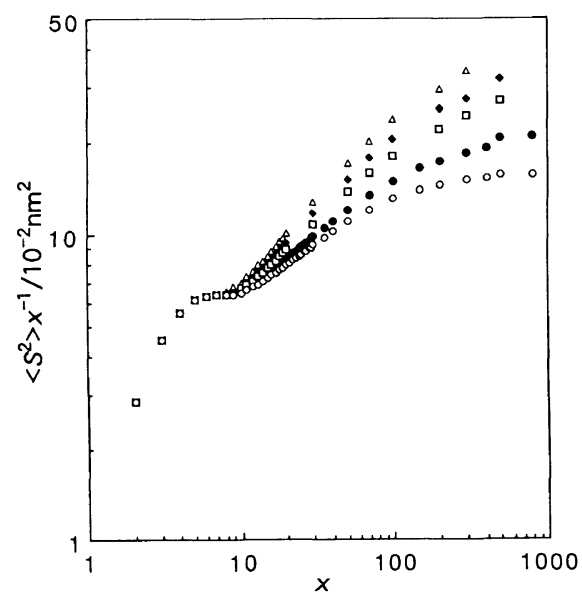

Figure 1. Simulation data of $\left\langle S^{2}\right\rangle / x$ plotted against $x$ for amylosic chains with $a=0(\bigcirc), 0.1 \mathrm{~nm}(\bigcirc), 0.2 \mathrm{~nm}$ $(\square), 0.3 \mathrm{~nm}(\bullet)$, and $0.4 \mathrm{~nm}(\triangle)$.

where $m_{i}$ denotes the mass of atom $i$ (numbered within the unit) and $s_{i}$, the distance between the atom $i$ and the mass center of the unit; the sum of $m_{i}$ over $i$ equals the molar mass of each glucose residue. The value of $\Delta$ should be about or slightly larger than $5 \times 10^{-16} \mathrm{~cm}^{2}$ (depending on the treatment of the hydrogen atoms of the hydroxyl groups), so that its neglect introduces considerable errors in $\left\langle S^{2}\right\rangle$ for $x$ smaller than 22 , i.e., for $M<3.6 \times 10^{3}$. However, no correction for $\Delta$ was made in this work, since $\left\langle S^{2}\right\rangle$ from the HW model parameters, to be compared with Monte Carlo $\left\langle S^{2}\right\rangle$ for such small $x$, is concerned only with the chain contour.

The Monte Carlo data of $\left\langle S^{2}\right\rangle$ obtained are shown in Figure 1. The ratio $\left\langle S^{2}\right\rangle / x$ for $a=0$ has a hump around $x=7$, reflecting the wellknown tendency toward helical loop formation $^{2,8,9}$ in the amylosic Monte Carlo chain. As $x$ increases, this ratio rises and almost levels off for $x>500$. Thus, the unperturbed chain is non-Gaussian at least up to $x=500$. For any nonzero $a,\left\langle S^{2}\right\rangle / x$ coincides with that for $a=0$ in the region of $x$ below 8 and begins to deviate from it when $x$ exceeds $8-11$. Such a critical $x$ for the onset of volume effect is perhaps too small. This may have something 
to do with the replacement of each glucose unit with a hard-core sphere. We note, however, that for $a=0.1 \mathrm{~nm}$, which is relevant to amylose in DMSO (see the analysis below), the volume effect stays rather small up to $x \approx 40$.

\section{DISCUSSION}

The mean-square radius of gyration of an $\mathrm{HW}$ bead chain with excluded volume $\mathrm{e}^{14-16}$ is determined by five parameters: $L$ (the contour length of the chain), $\lambda^{-1}$ (the stiffness parameter), $\kappa_{0}$ (the differential-geometrical curvature at the minimum energy of the chain), $\tau_{0}$ (the differential-geometrical torsion), and $B$ (the dimensionless parameter representing the strength of the excluded-volume interaction between a pair of beads). The first four parameters determine $\left\langle S^{2}\right\rangle_{0}$ (the value of $\left\langle S^{2}\right\rangle$ in the unperturbed state) or more generally any dimensional properties of an unperturbed HW chain. ${ }^{11}$ The last parameter (measured in units of $\lambda^{-1}$ ) is proportional to the binary cluster integral and related to the excluded-volume parameter $z$ by ${ }^{14-16}$

$$
z=(3 / 2 \pi)^{3 / 2} B(\lambda L)^{1 / 2}
$$

If $\lambda^{-1}$ is equal to the bead spacing, $z$ reduces to the familiar excluded-volume parameter in the conventional two-parameter theory. ${ }^{17}$ The contour length is related to $M$ by $L=M / M_{\mathrm{L}}$, with $M_{\mathrm{L}}$ being the molar mass per unit contour length.

Nakanishi et al. ${ }^{10}$ estimated $M_{L}, \lambda^{-1}, \kappa_{0} / \lambda$, and $\tau_{0} / \lambda$ for amylose in DMSO at $25^{\circ} \mathrm{C}$ to be $500 \mathrm{~nm}^{-1}, 4 \mathrm{~nm}, 3.5$, and 4 , respectively, from [ $\eta]$ data for $M<10^{4}$. This parameter set with $l=0.425 \mathrm{~nm}$ (instead of $0.44 \mathrm{~nm}$ ) gave $C_{\infty}=4.5$, but these authors corrected it to a value of 5 by referring to the experimental fact ${ }^{18}$ that the current polymer hydrodynamic theory ${ }^{12,17,19}$ somewhat overestimates the Flory viscosity factor $\left[\equiv[\eta] M /\left(6\left\langle S^{2}\right\rangle\right)^{3 / 2}\right]$ for long flexible chains in the unperturbed state. To diminish such a theoretical error, we here take $\lambda^{-1}$ to be $4.5 \mathrm{~nm}$ with $M_{\mathrm{L}}, \kappa_{0} / \lambda$, and $\tau_{0} / \lambda \mathrm{kept}$ at the above values; note that this $\lambda^{-1}$ value is hardly beyond the uncertainty of $\lambda^{-1}(4 \pm 0.5 \mathrm{~nm})^{10}$ originally estimated.

The experimental $\left\langle S^{2}\right\rangle$ of amylose in DMSO at $25^{\circ} \mathrm{C}^{10}$ varies in proportion to $M^{1.2}$. This exponent of 1.2 is the asymptotic value expected for long flexible chains in good solvents. ${ }^{19}$ Thus the remaining parameter $B$ was estimated from the $\left\langle S^{2}\right\rangle$ data and the HW parameter values $\left(M_{\mathrm{L}}=500 \mathrm{~nm}^{-1}, \lambda^{-1}=4.5\right.$ $\mathrm{nm}, \kappa_{0} / \lambda=3.5$, and $\left.\tau_{0} / \lambda=4\right)$ using the asymptotic relation ${ }^{20,21}$ between the radius expansion factor $\alpha_{\mathrm{s}}$ and $z$ :

$$
\alpha_{\mathrm{s}}^{2} \equiv\left\langle S^{2}\right\rangle /\left\langle S^{2}\right\rangle_{0}=1.53 z^{2 / 5} \quad(z \rightarrow \infty)
$$

Here, $\left\langle S^{2}\right\rangle_{0}$ of a long HW chain is given by ${ }^{11}$

$$
\begin{gathered}
\left\langle S^{2}\right\rangle_{0}=\left(4 \lambda^{2}+\tau_{0}^{2}\right) M /\left[6 \lambda M_{\mathrm{L}}\left(4 \lambda^{2}+\kappa_{0}^{2}+\tau_{0}^{2}\right)\right] \\
\left(\lambda M / M_{\mathrm{L}} \rightarrow \infty\right)
\end{gathered}
$$

The $B$ value obtained was 0.28 .

With the values for the HW parameters and $B$ together with the known expression for $\left\langle S^{2}\right\rangle_{0}$ of the HW chain with finite $\lambda M / M_{\mathrm{L}}$ (see eq 23 of ref 11), $\left\langle S^{2}\right\rangle$ was calculated as a function of $M$ from the combination ${ }^{15}$ of the Yamakawa-Stockmayer-Shimada perturbation theory ${ }^{14,16}$ for the HW bead chain (including the wormlike bead chain) and the Domb-Barrett equation $^{22}$ for flexible chains, i.e., from

$$
\begin{aligned}
\alpha_{\mathrm{s}}^{2}= & {\left[1+10 \tilde{z}+\left(\frac{70 \pi}{9}+\frac{10}{3}\right) \tilde{z}^{2}+8 \pi^{3 / 2} \tilde{z}^{3}\right]^{2 / 15} } \\
& \times\left[0.933+0.067 \exp \left(-0.85 \tilde{z}-1.39 \tilde{z}^{2}\right)\right]
\end{aligned}
$$

Here, $\tilde{z}$ is the scaled excluded-volume parameter defined by ${ }^{14-16}$

$$
\tilde{z}=(3 / 4) K z
$$

with $K$ being a known function of $\lambda L{ }^{16}$ In the coil limit, $\tilde{z}$ becomes identical to $z$ and eq 4 reduces to the original Domb-Barrett equation. ${ }^{22}$ We note that typical experimental data of $\alpha_{s}^{2}$ for flexible polymers ${ }^{23-25}$ are accurately described down to low $M$ by eq 4 with eq 5 . 


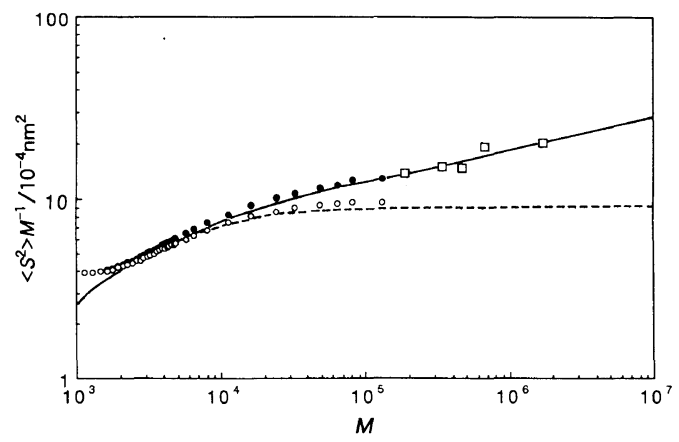

Figure 2. Simulation data of $\left\langle S^{2}\right\rangle / M$ for amylosic chains with $a=0.1 \mathrm{~nm}(\bigcirc)$ and $a=0(\bigcirc)$ compared with light scattering data ${ }^{10}(\square)$ for synthetic amylose samples in DMSO and calculated values for HW chains with $B=0.28(-)$ and $B=0(--)$. The $\mathrm{HW}$ model parameters determining $\left\langle S^{2}\right\rangle_{0}$ are $\lambda^{-1}=4.5 \mathrm{~nm}, M_{\mathrm{L}}=500 \mathrm{~nm}^{-1}$, $\kappa_{0} / \lambda=3.5$, and $\tau_{0} / \lambda=4.0$.

Figure 2 compares the Monte Carlo $\left\langle S^{2}\right\rangle / M$ values for $a=0.1 \mathrm{~nm}$ (the filled circles) with the experimental data ${ }^{10}$ in DMSO (the squares) and also with the calculated values for the perturbed HW chain with the above parameter set (the solid curve). The unfilled circles and the dashed line in the figure refer to the Monte Carlo $\left\langle S^{2}\right\rangle_{0}$ (for $a=0$ ) and the calculated $\left\langle S^{2}\right\rangle_{0}$ for the unperturbed HW chain $(B=0)$, respectively. It can be seen that the simulation data for $a=0.1 \mathrm{~nm}$ are connected smoothly with the measured values in DMSO and that both are fitted closely by the solid curve for $M>2 \times 10^{3}$, i.e., for $x>12$. In this $M$ range, the simulation data without excluded volume also come close to the dashed line. The latter is based entirely on the $[\eta]$ data of Nakanishi et al. for $M<10^{4}$, for which excluded-volume effects on $\left\langle S^{2}\right\rangle$ are seen to be practically insignificant. Thus, it may be concluded that the present simulation data (for $M>2 \times 10^{3}$ ) are consistent with the measured $[\eta]$ and $\left\langle S^{2}\right\rangle$ in DMSO. In other words, some irregular helical conformation, which has long been suspected for amylose in solution on the basis of conformation analysis, must be similar to that deduced from dilute-solution data with the aid of the HW model. For $M$ below $2 \times 10^{3}$, the simulation data (with or without excluded volume) deviate appreciably from the solid line. Although this deviation ought to be seen by further study, it is attributable, to a large extent, to the length scale adopted by Nakanishi et al. in the analysis of the viscosity data, as explained below.

Yamakawa and coworkers ${ }^{26}$ found that the Monte Carlo data of Jordan et al. ${ }^{8}$ for the persistence vector and $\left\langle R^{2}\right\rangle$ (without excluded volume) are accurately reproduced by $\mathrm{HW}$ theoretical curves (for an appropriately chosen set of parameters) over the entire possible range of $x(\geq 2)$ when the HW chain contour is taken along the helical sequence. The chain contour so chosen corresponds to a small length scale of one glucose residue. On the other hand, if the contour is taken along the helix axis, i.e., if a large length scale of a few glucose units (relevant to analysis of data for steady-state transport coefficients) ${ }^{26}$ is adopted, the HW thoretical curve for $\left\langle R^{2}\right\rangle$ deviates downward from the Monte Carlo data at $x$ smaller than 10 (see Figure 9 of ref 26). The analysis of $[\eta]$ made by Nakanishi et al. indeed refers to such a large length scale of two to four glucose residues.

\section{REFERENCES}

1. W. Burchard, Makromol. Chem., 59, 16 (1963).

2. S. Kitamura and T. Kuge, Food Hydrocoll., 3, 313 (1989).

3. W. Banks and C. T. Greenwood, "Starch and its Components," Edinburgh University Press, Edinburgh, 1975.

4. S. G. Ring, K. J. I'Anson, and V. J. Morris, Macromolecules, 18, 182 (1985).

5. T. Norisuye, to appear in Food Hydrocoll.

6. M. Fujii, K. Honda, and H. Fujita, Biopolymers, 12, 1177 (1973).

7. V. S. Rao, N. Yathindra, and P. R. Sundararajan, Biopolymers, 8, 325 (1969).

8. R. C. Jordan, D. A. Brant, and A. Cesàro, Biopolymers, 17, 2617 (1978).

9. S. Kitamura, T. Okamoto, Y. Nakata, T. Hayashi, and T. Kuge, Biopolymers, 26, 537 (1987).

10. Y. Nakanishi, T. Norisuye, A. Teramoto, and S. Kitamura, Macromolecules, 26, 4220 (1993). 
11. H. Yamakawa, in "Molecular Conformation and Dynamics of Macromolecules in Condensed Systems," M. Nagasawa, Ed., Elsevier, Amsterdam, 1988, p 21.

12. T. Yoshizaki, I. Nitta, and H. Yamakawa, Macromolecules, 21, 165 (1988).

13. F. T. Wall and J. J. Erpenbeck, J. Chem. Phys., 30, 634 (1959).

14. H. Yamakawa and W. H. Stockmayer, J. Chem. Phys., 57, 2843 (1972).

15. H. Yamakawa and J. Shimada, J. Chem. Phys., 83, 2607 (1985).

16. J. Shimada and H. Yamakawa, J. Chem. Phys., 85, 591 (1986).

17. H. Yamakawa, "Modern Theory of Polymer Solutions," Harper \& Row, New York, 1971.

18. T. Konishi, T. Yoshizaki, and H. Yamakawa, Macromolecules, 24, 5614 (1991).
19. H. Fujita, "Polymer Solutions," Elsevier, Amsterdam, 1990.

20. M. Lax, A. J. Barrett, and C. Domb, J. Phys. A: Math. Gen., 11, 361 (1978); A. J. Barrett and C. Domb, Proc. Roy. Soc. London, Ser. A, 376, 361 (1981).

21. Y. Miyaki, Y. Einaga, and H. Fujita, Macromolecules, 11, 1180 (1978).

22. C. Domb and A. J. Barrett, Polymer, 17, 179 (1976).

23. T. Kitagawa, J. Sadanobu, and T. Norisuye, Macromolecules, 23, 602 (1990).

24. F. Abe, Y. Einaga, T. Yoshizaki, and H. Yamakawa, Macromolecules, 26, 1184 (1993).

25. F. Abe, K. Horita, Y. Einaga, and H. Yamakawa, Macromolecules, in press.

26. M. Fujii, K. Nagasaka, J. Shimada, and H. Yamakawa, Macromolecules, 16, 1613 (1983). 